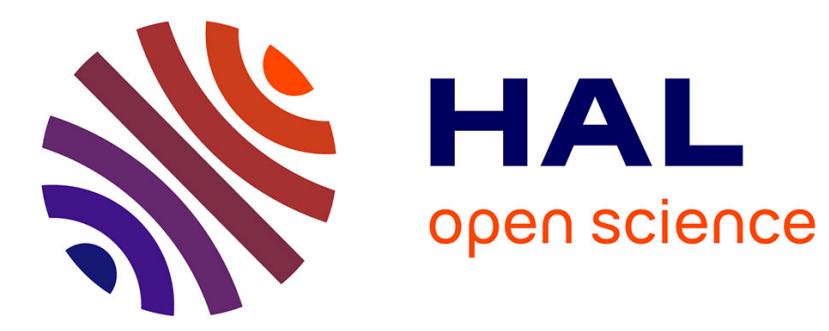

\title{
Open-loop control of absolutely unstable domains Benoît Pier
}

\section{To cite this version:}

Benoit Pier. Open-loop control of absolutely unstable domains. Proceedings of the Royal Society A: Mathematical, Physical and Engineering Sciences, 2003, 459, pp.1105-1115. 10.1098/rspa.2002.1086 . hal-00120027

\section{HAL Id: hal-00120027 https://hal.science/hal-00120027}

Submitted on 13 Dec 2006

HAL is a multi-disciplinary open access archive for the deposit and dissemination of scientific research documents, whether they are published or not. The documents may come from teaching and research institutions in France or abroad, or from public or private research centers.
L'archive ouverte pluridisciplinaire HAL, est destinée au dépôt et à la diffusion de documents scientifiques de niveau recherche, publiés ou non, émanant des établissements d'enseignement et de recherche français ou étrangers, des laboratoires publics ou privés. 


\title{
Open-loop control of absolutely unstable domains
}

\author{
By BENOÎT PIER \\ Department of Applied Mathematics and Theoretical Physics, \\ University of Cambridge, Silver Street, Cambridge CB3 9EW, UK
}

Spatially varying systems with a central absolutely unstable region are known to give rise to self-sustained finite-amplitude globally synchronized structures. The present investigation shows how such an instrinsic behaviour may be controlled by small-amplitude forcing applied upstream of the fully developed oscillations. This technique allows the tuning of the frequency of the entire system to any frequency in a wide range, exspending only an exponentially small power.

Keywords: Absolute instability, Self-sustained oscillations, Control

\section{Introduction}

Self-sustained finite-amplitude oscillations are produced in a great variety of situations ranging from fluid flows (Huerre 2000), chemical reactions (Kapral \& Showalter 1995) and biological processes (Winfree 1987) to solar activity cycles (Meunier et al. 1997). Such an intrinsic behaviour is often unwanted, e.g., it induces structural damage in flows around obstacles or affects performance of turbocompressors. Understanding the mechanism responsible for these oscillations may then suggest methods to suppress or to control them. In many situations (e.g. three-dimensional boundary layers, Reed \& Saric 1989) the naturally occurring nonlinear waves are prone to secondary instabilities which in turn lead to a turbulent régime. Hence a controlled modification of the primary nonlinear state is desirable if one wants to delay (or possibly to enhance) the onset of turbulence. Although very efficient, optimal or robust control theory (Bewley \& Liu 1998) is difficult to implement since it relies on heavy numerical computations and on a precise knowledge of the system state. Our objective is to devise a control strategy applicable to a broad class of systems that takes advantage of the global instability mechanism and requires only extremely weak localized action.

In the context of spatially varying systems, the existence of intrinsic oscillations (Huerre \& Monkewitz 1990; Pier et al. 2001) is closely related to the transition from convective to absolute instability, as determined by the complex local absolute frequency $\omega_{0}(X)$ (Briggs 1964; Bers 1983). In stable or convectively unstable (CU) regions, characterized by $\omega_{0, i}(X) \equiv \operatorname{Im} \omega_{0}(X)<0$, perturbations either decay or are swept away by advection. Convectively unstable systems thus display no instrinsic behaviour and essentially behave as amplifiers of external noise: without continuous external input the medium returns to its unperturbed state. By contrast, in absolutely unstable (AU) regions where $\omega_{0, i}(X)>0$, perturbations grow in situ and hence may lead to non trivial dynamics without external forcing. It has 
been shown that the onset of global nonlinear oscillations in infinite systems occurs as soon as local absolute instability is reached somewhere in the medium (Pier et al. 2001). Other varieties of nonlinear global modes have been obtained in semi-infinite systems (Couairon \& Chomaz 1996, 1997a,b, 1999) which are not discussed here.

The sensitivity of $\mathrm{CU}$ open flows to external perturbations has been well established, e.g., for free shear layers (Ho \& Huerre 1984), the Taylor-Couette flow with through flow (Babcock et al. 1991) or the wake behind a cylinder (Le Gal \& Croquette 2000). In the AU régime similar systems generally display robust natural oscillations that are believed to be insensitive to low noise levels (Huerre 2000). However, a variety of unstable systems (e.g. side-branching dendrites, Bouissou et al. 1990) are known to exhibit a strong receptivity to periodic rather than random perturbations. Similarly, by revisiting spatially developing media displaying a central AU domain embedded in two semi-infinite at most $\mathrm{CU}$ regions, the present investigation shows that small-amplitude harmonic forcing can completely modify the natural nonlinear behaviour. It should be emphasized that the aim is not to suppress the fluctuations but to tune them to an externally imposed frequency and thereby also to modify the local wavelengths and amplitudes.

\section{Problem formulation}

The present study is based on a general one-dimensional model equation that accounts for the dynamics of a variety of physical systems, is tractable by analytical methods and has a similar form than the celebrated Ginzburg-Landau type equations (Cross \& Hohenberg 1993). In the past already, the model (2.1) has on many occasions proven a convenient testground to recognize and study generic features that have later been identified in a variety of situations. The same strategy is adopted here. We assume that the system under consideration is described by a complex scalar field $\psi(x, t)$ in an infinite one-dimensional spatially inhomogenous domain and is governed by

$$
\begin{aligned}
\frac{\partial \psi}{\partial t}= & -\mathrm{i}\left(\omega_{0}(X)+\frac{1}{2} \omega_{k k}(X) k_{0}(X)^{2}\right) \psi+\omega_{k k}(X) k_{0}(X) \frac{\partial \psi}{\partial x} \\
& +\frac{\mathrm{i}}{2} \omega_{k k}(X) \frac{\partial^{2} \psi}{\partial x^{2}}-\mathrm{i} \gamma(X)|\psi|^{2} \psi+S(x, t),
\end{aligned}
$$

where the complex functions $\omega_{0}(X), k_{0}(X), \omega_{k k}(X)$ and $\gamma(X)$ account for the local properties of the medium and only depend on a slow space variable $X=\epsilon x$. The weak inhomogeneity parameter $\epsilon \ll 1$ is defined as the ratio of the typical instability length scale to the non-uniformity length scale of the medium. The source function $S(x, t)$ represents an externally applied forcing to be used below.

In the subsequent discussion, constant use is made of the local linear and nonlinear properties of system (2.1), which are presented now. Local characteristics are derived from (2.1) by freezing $X$ and studying the corresponding spatially homogenous medium. Linear properties pertain to the dynamics of small-amplitude normal modes of the form $\mathrm{e}^{\mathrm{i}(k x-\omega t)}$ where the complex frequency $\omega$ and complex wavenumber $k$ satisfy the linear dispersion relation

$$
\omega=\Omega^{\ell}(k, X) \equiv \omega_{0}(X)+\frac{1}{2} \omega_{k k}(X)\left(k-k_{0}(X)\right)^{2} .
$$


The particular form in which the coefficients of (2.1) have been cast brings to the fore the local complex absolute frequency $\omega_{0}(X)$ associated with the local complex absolute wavenumber $k_{0}(X)$ determined by a zero group velocity condition as (Briggs 1964; Bers 1983)

$$
\omega_{0}(X)=\Omega^{\ell}\left(k_{0}(X), X\right) \quad \text { with } \quad \frac{\partial \Omega^{\ell}}{\partial k}\left(k_{0}(X), X\right)=0 .
$$

In a typical situation of interest, the local absolute growth rate $\omega_{0, i}(X)$ displays a single maximum $\omega_{0, i}^{\max }$ and the medium is stable for large $|X|$. The system under consideration then exhibits an AU domain whenever $\omega_{0, i}^{\max }>0$.

The model equation (2.1) also admits finite-amplitude travelling waves $R \mathrm{e}^{\mathrm{i}(k x-\omega t)}$ where the real amplitude $R$, real frequency $\omega$ and real wavenumber $k$ satisfy the nonlinear dispersion relations

$$
\begin{aligned}
\omega=\Omega^{n \ell}(k, X) & \equiv \frac{\operatorname{Im}\left[\gamma(X)^{\star} \Omega^{\ell}(k, X)\right]}{-\gamma_{i}(X)} \\
R^{2}=\mathcal{R}(k, X)^{2} & \equiv \frac{\Omega_{i}^{\ell}(k, X)}{-\gamma_{i}(X)}
\end{aligned}
$$

with superscript $\star$ denoting complex conjugate. Note that the condition of stabilizing nonlinearities requires that $\gamma_{i}(X)<0$. At a given value of $X$, nonlinear wavetrains thus exist $\left(R^{2}>0\right)$ for the range of real wavenumbers $k$ associated with a positive temporal growth rate $\Omega_{i}^{\ell}(k, X)>0$ and the nonlinear amplitude $R$ vanishes for marginal wavenumbers associated with $\Omega_{i}^{\ell}(k, X)=0$.

\section{Signalling problem}

Of particular importance to the present work is the spatial response to localized time-harmonic forcing switched on at $t=0$. This problem is governed by equation (2.1) with the source term

$$
S(x, t)=A_{f} \delta\left(x-x_{f}\right) H(t) \mathrm{e}^{-\mathrm{i} \omega_{f} t},
$$

where $A_{f}, X_{f} \equiv \epsilon x_{f}$ and $\omega_{f}$ represent the forcing amplitude, location and (real) frequency respectively, $\delta$ denotes the Dirac delta function $\dagger$ and $H$ the Heaviside unit step function.

Consider the spatial response of system (2.1) in a situation where no AU region is present, i.e., $\omega_{0, i}^{\max }<0$. Then no self-sustained oscillations are produced and the resulting field $\psi_{f}(x, t)$ is purely due to the external forcing (figure 1$)$. Under the slowly varying medium hypothesis $(\epsilon \ll 1)$, the long-time response of constant frequency $\omega_{f}$ is obtained in terms of Werner-Kramers-Brillouin (WKB) asymptotic approximations (Bender \& Orszag 1978) of the form

$$
\psi_{f}=A\left(X ; \omega_{f}\right) \exp \left(\frac{\mathrm{i}}{\epsilon} \int_{X_{f}}^{X} k\left(u ; \omega_{f}\right) \mathrm{d} u-\mathrm{i} \omega_{f} t\right) .
$$

$\dagger$ The representation $\delta(x)=\frac{1}{\sqrt{\pi} \sigma} \exp \left(-x^{2} / \sigma^{2}\right)$ with $\sigma=1 / 4$ is used in the numerical implementation below. 


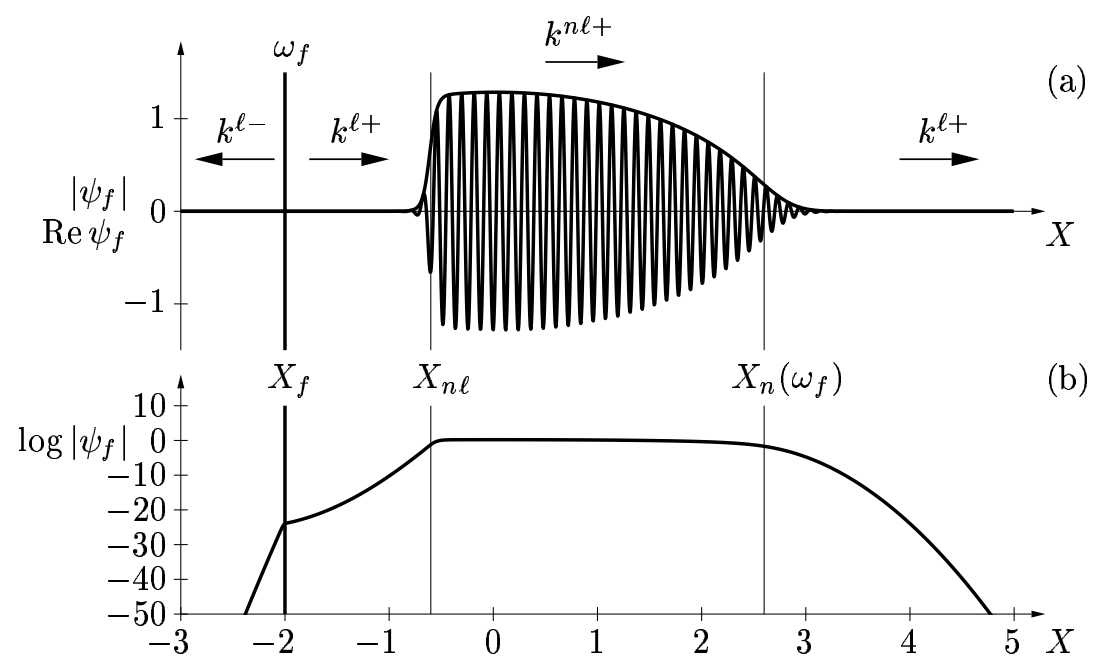

Figure 1. Envelope $\left|\psi_{f}\right|$ and real part $\operatorname{Re} \psi_{f}$ of spatial response of governing equation to time-harmonic forcing of frequency $\omega_{f}=1$ and amplitude $A_{f}=10^{-10}$ applied at $X_{f}=-2$. Numerical simulation performed with $\omega_{0}=0.4-0.2\left(1+X^{2}\right) \mathrm{i}, k_{0}=1-1.5 \mathrm{i}, \omega_{k k}=1-0.5 \mathrm{i}$, $\gamma=0.2-\mathrm{i}$ and $\epsilon=1 / 25$. Decaying upstream response follows $k^{\ell-}\left(X ; \omega_{f}\right)$ branch. Downstream response exponentially grows according to $k^{\ell+}\left(X ; \omega_{f}\right)$ to reach finite amplitude at $X_{n \ell}=-0.6$ where the nonlinear $k^{n \ell+}\left(X ; \omega_{f}\right)$ branch takes over. Further downstream, beyond the neutral station $X_{n}\left(\omega_{f}\right)=2.6$, the decaying $k^{\ell+}\left(X ; \omega_{f}\right)$ prevails.

In this expression, the local wavenumber branch $k\left(X ; \omega_{f}\right)$ accounts for the fast spatial variations whereas the amplitude function $A\left(X ; \omega_{f}\right)$ only depends on slow space and is obtained in classical WKB fashion as a series expansion

$$
A\left(X ; \omega_{f}\right) \sim A_{0}\left(X ; \omega_{f}\right)+\epsilon A_{1}\left(X ; \omega_{f}\right)+\epsilon^{2} A_{2}\left(X ; \omega_{f}\right)+\ldots
$$

For reasons that become clear below, we only consider exponentially small forcing amplitudes $A_{f}=\mathrm{e}^{\alpha / \epsilon}$ with $\alpha<0$. In the vicinity of the forcing location $X_{f}$, the spatial response is then governed by linear dynamics and the resulting complex local wavenumber branch is obtained by solving (2.2) for $\omega=\omega_{f}$ as

$$
k^{\ell \pm}\left(X ; \omega_{f}\right)=k_{0}(X) \pm \sqrt{2 \frac{\omega_{f}-\omega_{0}(X)}{\omega_{k k}(X)}} .
$$

Causality considerations (Briggs 1964; Bers 1983) allow the unambiguous definition of the square-root branches in (3.4) such that the $k^{\ell+}\left(k^{\ell-}\right)$ spatial branch pertains to the downstream $X>X_{f}$ (upstream $X<X_{f}$ ) side of the forcing location. Upon substituting (3.4) with (3.2) into the governing equation (2.1), a solvability condition governing the leading-order amplitude is derived as

$$
\frac{\mathrm{d} A_{0}}{\mathrm{~d} X}=-\frac{1}{2} \frac{\mathrm{d} k^{\ell \pm} / \mathrm{d} X}{k^{\ell \pm}\left(X ; \omega_{f}\right)-k_{0}(X)} A_{0}\left(X ; \omega_{f}\right) .
$$

Invoking continuity of the response at $X=X_{f}$, the exact long-time linear response is then obtained to leading order as

$$
A_{0}\left(X ; \omega_{f}\right)=A_{f} C_{f} \exp \left(\int_{X_{f}}^{X}-\frac{1}{2} \frac{\mathrm{d} k^{\ell \pm} / \mathrm{d} u}{k^{\ell \pm}\left(u ; \omega_{f}\right)-k_{0}(u)} \mathrm{d} u\right)
$$


with

$$
A_{f}=\exp \frac{\alpha}{\epsilon} \quad \text { and } \quad C_{f}=\frac{2}{\omega_{k k}\left(X_{f}\right)\left[k^{\ell+}\left(X_{f} ; \omega_{f}\right)-k^{\ell-}\left(X_{f} ; \omega_{f}\right)\right]}
$$

The modulus $\left|\psi_{f}\right|$ of the forced response (3.2) in the linear regions is then derived to leading order as

$$
\log \left|\psi_{f}\right| \sim \frac{1}{\epsilon}\left(\alpha-\int_{X_{f}}^{X} k_{i}^{\ell \pm}\left(u ; \omega_{f}\right) \mathrm{d} u\right) .
$$

In stable or at most $\mathrm{CU}$ regions the upstream spatial response decays for all frequencies, $k_{i}^{\ell-}(X ; \omega)<0$. The linear approximation then applies to the entire region $-\infty<X<X_{f}$ upstream of the forcing location. For forcing applied at a CU location, there exists a range of frequencies associated with downstream growth, i.e., such that $k_{i}^{\ell+}\left(X_{f} ; \omega_{f}\right)<0$. The downstream spatial response then exponentially grows to reach finite amplitude levels. Nonlinear saturation prevents further growth and leads to a nonlinear wavetrain at the forcing frequency. The nonlinear saturation station $X_{n \ell}$ where the modulus $\left|\psi_{f}\right|$ takes $\mathcal{O}(1)$ values depends on the forcing amplitude and is determined by (3.8) as

$$
\int_{X_{f}}^{X_{n \ell}} k_{i}^{\ell+}\left(u ; \omega_{f}\right) \mathrm{d} u=\alpha .
$$

Thus the downstream linear $k^{\ell+}\left(X ; \omega_{f}\right)$ spatial branch prevails in the interval $X_{f}<$ $X<X_{n \ell}$ which is of finite extent in terms of the slow variable $X$ only for an exponentially weak forcing amplitude $A_{f}=\mathrm{e}^{-|\alpha| / \epsilon}$ with $|\alpha|=\mathcal{O}\left(\epsilon^{0}\right)$.

Downstream of $X_{n \ell}$, the spatial response is made up of a finite-amplitude saturated wavetrain of frequency $\omega_{f}$ governed by the local nonlinear dispersion relations $(2.3,2.4)$. The local wavenumber in $(3.2)$ then follows the real spatial branch $k^{n \ell+}\left(X ; \omega_{f}\right)$ obtained by solving $(2.3)$ for the prescribed forcing frequency $\omega=\omega_{f}$. In the nonlinear régime, the modulus of the leading-order amplitude (3.3) is determined by (2.4) as

$$
\left|A_{0}\left(X ; \omega_{f}\right)\right|=\mathcal{R}\left(k^{n \ell+}\left(X ; \omega_{f}\right), X\right),
$$

whereas its phase is governed by a solvability condition at higher order. A detailed discussion of the relationship between nonlinear spatial response and causality as well as a full derivation of nonlinear WKB approximations can be found in (Pier et al. 2001).

The nonlinear wavetrain of local wavenumber $k^{n \ell+}\left(X ; \omega_{f}\right)$ prevails in the interval $X_{n \ell}<X<X_{n}\left(\omega_{f}\right)$ beyond which the spatial response exponentially decays. The transition station $X_{n}\left(\omega_{f}\right)$ from a nonlinear to a linear régime is characterized by the neutral stability condition $\Omega_{i}^{\ell}\left(k^{n \ell+}\left(X_{n} ; \omega_{f}\right), X_{n}\right)=0$. The amplitude of the nonlinear travelling wave vanishes there and the complex branch $k^{\ell+}\left(X ; \omega_{f}\right)$ continuously takes over in the downstream linear region $X>X_{n}\left(\omega_{f}\right)$. It should be noted that for a given forcing frequency the onset station of nonlinearities $X_{n \ell}$ also depends on the forcing amplitude whereas the neutral station $X_{n}$ does not. 


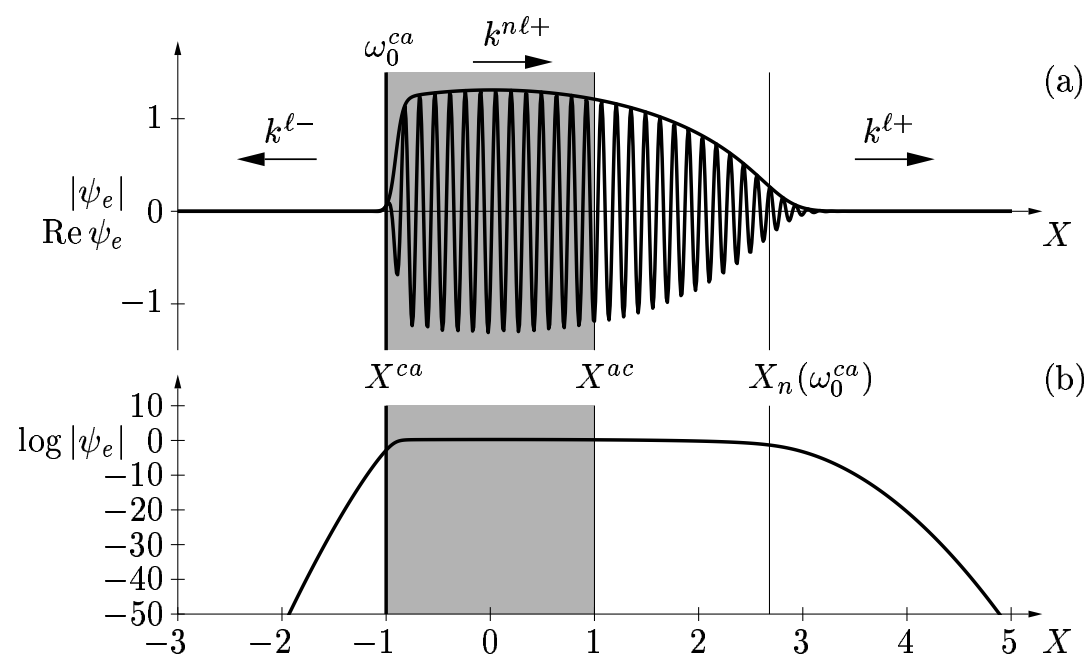

Figure 2. Envelope $\left|\psi_{e}\right|$ and real part $\operatorname{Re} \psi_{e}$ of self-sustained structure illustrated by numerical simulation of unforced governing equation with $\omega_{0}=0.4+0.3\left(1-X^{2}\right) \mathrm{i}, k_{0}=1-1.5 \mathrm{i}$, $\omega_{k k}=1-0.5 \mathrm{i}, \gamma=0.2-\mathrm{i}$ and $\epsilon=1 / 25$. Central AU domain $X^{c a}=-1<X<X^{a c}=1$ is indicated in grey. The front of frequency $\omega_{0}^{c a}=0.4$ at $X^{c a}$ generates the upstream linear $k^{\ell-}\left(X ; \omega_{0}^{c a}\right)$ branch and the downstream nonlinear $k^{n \ell+}\left(X ; \omega_{0}^{c a}\right)$ branch. Further downstream, beyond the neutral station $X_{n}\left(\omega_{0}^{c a}\right)=2.8$, the exponentially decaying $k^{\ell+}\left(X ; \omega_{0}^{c a}\right)$ prevails.

\section{Self-sustained oscillations}

Before applying the above results to control media displaying a central AU region, their self-sustained behaviour is summarized. Consider a situation where $\omega_{0, i}^{\max }>0$, associated with an AU interval $X^{c a}<X<X^{a c}$. Then a fully nonlinear temporally periodic state is reached without external input (Pier et al. 2001). At $\omega_{0, i}^{\max }=0$ onset of a self-sustained state occurs via a saddle-node bifurcation; for moderate values of $\omega_{0, i}^{\max }>0$ an 'elephant' or 'steep' nonlinear structure (Pier \& Huerre 2001) is selected (figure 2), characterized by a sharp (Dee \& Langer 1983) front at the upstream boundary $X^{c a}$ of the AU domain. This front of real frequency $\omega_{0}^{c a} \equiv \omega_{0}\left(X^{c a}\right)$ acts as a wavemaker, hence tuning the entire system to a single frequency. The entire field $\psi_{e}(x, t)$ may then be interpreted as the spatial response to this front. Indeed, in the upstream linear region $X<X^{c a}$, the complex field $\psi_{e}(x, t)$ follows the complex spatial $k^{\ell-}\left(X ; \omega_{0}^{c a}\right)$ branch and its modulus exponentially decays according to

$$
\log \left|\psi_{e}\right| \sim-\frac{1}{\epsilon} \int_{X^{c a}}^{X} k_{i}^{\ell-}\left(u ; \omega_{0}^{c a}\right) \mathrm{d} u .
$$

The region $X^{c a}<X<X_{n}\left(\omega_{0}^{c a}\right)$ is made up of finite-amplitude waves following the nonlinear $k^{n \ell+}\left(X ; \omega_{0}^{c a}\right)$ branch. Beyond the neutrally stable station $X_{n}\left(\omega_{0}^{c a}\right)$, the decaying linear $k^{\ell+}\left(X ; \omega_{0}^{c a}\right)$ branch prevails. Further above onset of a nonlinear selfsustained state, i.e., for larger values of $\omega_{0, i}^{\max }$, 'steep' modes may give way to 'soft' modes (Pier et al. 2001; Pier \& Huerre 2001); their control is not discussed here.

We emphasize the important fact that the fully nonlinear self-sustained structure is determined by a purely linear criterion based only on the local absolute frequency curve $\omega_{0}(X)$. The front is of 'pulled' (Ebert \& Saarloos 2000) type and its dynamics is determined by the decaying tail: the front envelope is stationary in time if the 
front location is precisely at the CU/AU transition station $X^{c a}$. Note also that the region $X>X^{a c}$ downstream of the AU domain is slaved to the wavemaker prevailing at $X^{c a}$ and does not play an active part in the dynamics. The result that self-sustained nonlinear oscillations are triggered by a stationary front at $X^{c a}$ suggests that this instrinsic behaviour may be modified by perturbing the front.

\section{Control}

Consider now applying to the intrinsic nonlinear structure a forcing of frequency $\omega_{f}$ and small amplitude $A_{f}=\mathrm{e}^{\alpha / \epsilon}$ localized at $X_{f}$ in the CU region, $X_{f}<X^{c a}$. For small forcing amplitudes, both the spatial response of frequency $\omega_{f}$ and the selfsustained mode of frequency $\omega_{0}^{c a}$ are governed by linear dynamics in the neighbourhood of $X_{f}$. The resulting field in the linear region is then obtained as a superposition $\psi=\psi_{f}+\psi_{e}$ of the forced response of frequency $\omega_{f}$ and the natural oscillations of frequency $\omega_{0}^{c a}$. At a given location, the dominant component in the total field depends on the relative modulus of $\psi_{f}(3.8)$ and $\psi_{e}$ (4.1).

In the interval $X_{f}<X<X^{c a}$, the local spatial growth rates of $\psi_{f}$ and $\psi_{e}$ are respectively given by $-k_{i}^{\ell+}\left(X ; \omega_{f}\right)$ and $-k_{i}^{\ell-}\left(X ; \omega_{0}^{c a}\right)$. From (3.4) and causality it is seen that always $k_{i}^{\ell+}(X ; \omega)>k_{i}^{\ell-}(X ; \omega)$, hence the result

$$
k_{i}^{\ell+}\left(X ; \omega_{f}\right)>k_{i}^{\ell-}\left(X ; \omega_{0}^{c a}\right)
$$

holds for forcing frequencies $\omega_{f}$ sufficiently close to the natural frequency $\omega_{0}^{c a}$. A comprehensive survey of all possible configurations of equation (2.1) has not been attempted, but inequality (5.1) was found to hold for all forcing frequencies associated with downstream growth. The result (5.1) means that from the forcing location $X_{f}$ towards the front location $X^{c a}$ the modulus of the natural oscillations $\left|\psi_{e}\right|$ grows faster than the forced response $\left|\psi_{f}\right|$. The regions dominated by either the forcing frequency $\omega_{f}$ or the natural frequency $\omega_{0}^{c a}$ may then be monitored for different values of the forcing amplitude $\mathrm{e}^{\alpha / \epsilon}$ while keeping the forcing location and frequency at prescribed values (figure 3 ).

For extremely weak forcing $\alpha<\alpha_{0}$ with

$$
\alpha_{0} \equiv \int_{X_{f}}^{X^{c a}} k_{i}^{\ell-}\left(u ; \omega_{0}^{c a}\right) \mathrm{d} u<0
$$

the forced response is dominated by the intrinsic oscillations at the very forcing location. Due to the different spatial growth rates (5.1), the self-sustained mode of frequency $\omega_{0}^{c a}$ then prevails over the forced response in the entire system.

For higher forcing amplitudes $\alpha>\alpha_{0}$, the response $\psi_{f}$ dominates $\psi_{e}$ at the forcing location $X_{f}$. However, since $\left|\psi_{e}\right|$ grows faster than $\left|\psi_{f}\right|$ with increasing $X$, the forcing frequency $\omega_{f}$ only prevails in the interval $X_{f}<X<X_{b}$ extending towards the location $X_{b}$ where both components display a similar modulus and beyond which $\psi_{e}$ prevails. The boundary $X_{b}$ between the regions tuned to the forcing frequency $\omega_{f}$ or to the natural frequency $\omega_{0}^{c a}$ is thus determined as

$$
\int_{X_{f}}^{X_{b}} k_{i}^{\ell+}\left(u ; \omega_{f}\right) \mathrm{d} u-\int_{X_{b}}^{X^{c a}} k_{i}^{\ell-}\left(u ; \omega_{0}^{c a}\right) \mathrm{d} u=\alpha .
$$




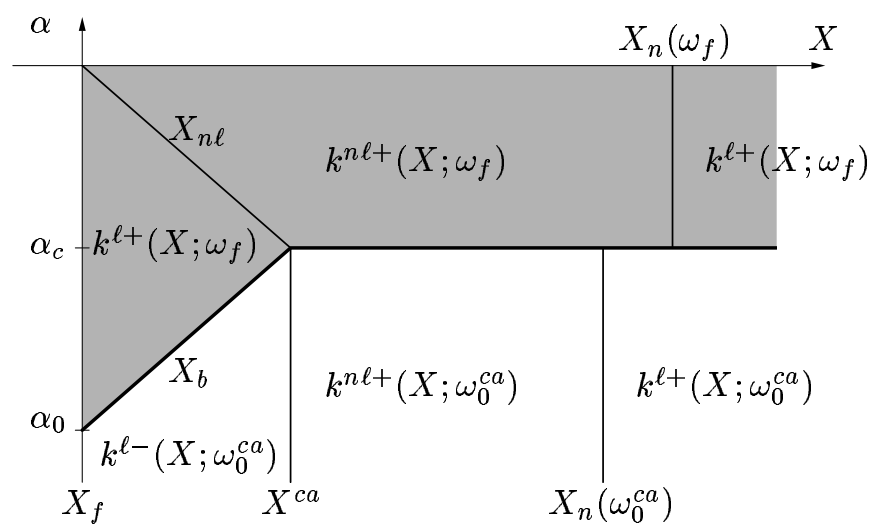

Figure 3. Map of regions tuned to forcing frequency $\omega_{f}$ (shaded) or to intrinsic frequency $\omega_{0}^{c a}$ as a function of the forcing amplitude $A_{f}=\mathrm{e}^{\alpha / \epsilon}$ while forcing frequency $\omega_{f}$ and location $X_{f}$ are kept constant. Below the control threshold $\alpha<\alpha_{c}$, the forcing frequency $\omega_{f}$ prevails in the region $X_{f}<X<X_{b}$ whereas the self-sustained structure of frequency $\omega_{0}^{c a}$ survives in $X>X_{b}$. For $\alpha>\alpha_{c}$, the forced response supersedes the instrinsic oscillations in the entire system. At each $\alpha$, the dominant component of the total field $\psi$ follows the spatial linear or nonlinear branches indicated in the corresponding regions.

With increasing forcing level, the region dominated by $\omega_{f}$ grows in size and the boundary $X_{b}$ eventually reaches the front location $X^{c a}$. This occurs at the critical forcing amplitude $\alpha_{c}$ given by

$$
\alpha_{c} \equiv \int_{X_{f}}^{X^{c a}} k_{i}^{\ell+}\left(u ; \omega_{f}\right) \mathrm{d} u
$$

For forcing levels in the range $\alpha_{0}<\alpha<\alpha_{c}$, the response of frequency $\omega_{f}$ does not achieve $\mathcal{O}(1)$ amplitudes at the front location $X^{c a}$ and is thus unable to perturb the nonlinear self-sustained waves of frequency $\omega_{0}^{c a}$ prevailing for $X>X^{c a}$ (figure 4a). For $\alpha=\alpha_{c}$ however, the forced response reaches finite amplitude precisely at $X^{c a}$ and competes with the stationary front of the intrinsic nonlinear structure. Numerical simulations performed in this situation (figure $4 \mathrm{~b}$ ) reveal that the forced response is then able to continually perturb the natural oscillations and the system does not converge to an equilibrium state tuned at a single frequency.

For slightly stronger forcing $\alpha>\alpha_{c}$, the response at frequency $\omega_{f}$ reaches nonlinear saturation upstream of the front, i.e., $X_{n \ell}<X^{c a}$. In this régime (figure 4c), the intrinsic oscillations at $\omega_{0}^{\text {ca }}$ are completely suppressed and replaced by the forced response in the entire domain. For still higher forcing amplitudes, the system remains tuned at $\omega_{f}$ and its spatial structure does not evolve except that the nonlinear saturation station $X_{n \ell}$ moves upstream towards $X_{f}$ (cf. figure 3 ).

This behaviour may be interpreted as the result of two competing sources of different frequencies at different locations: the self-sustained $\omega_{0}^{c a}$-front at $X^{c a}$ responsible for the natural nonlinear structure and the external $\omega_{f}$-forcing at $X_{f}$. In the absence of external forcing the front at $X^{c a}$ acts as a cornerstone upon which the global structure is based; this front dictates its frequency to the entire system and generates the downstream developing nonlinear wavetrain covering the AU region. When forcing is applied at $X_{f}$, the intrinsic wavemaker at $X^{c a}$ survives only 


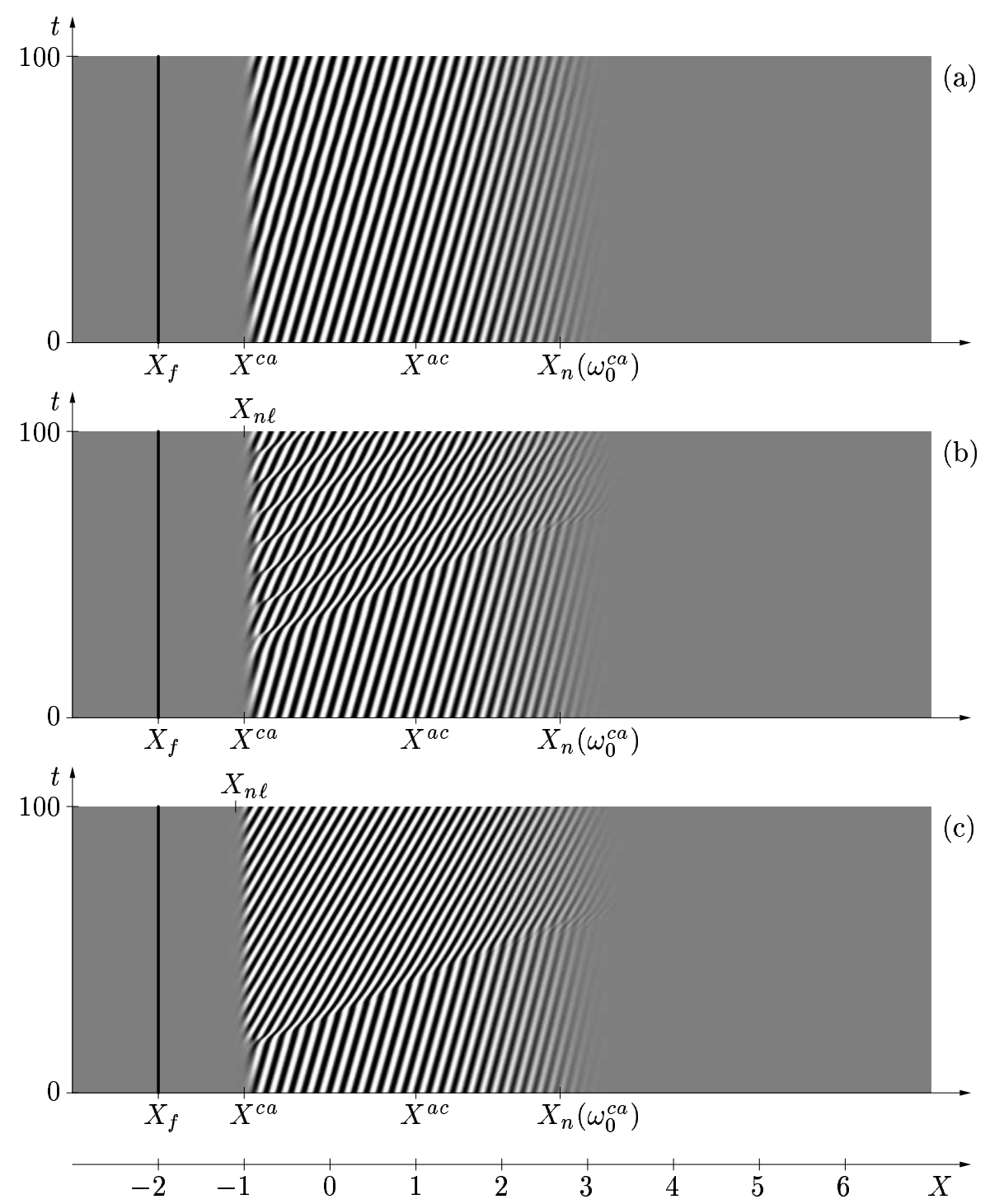

Figure 4. Control of self-sustained structure by localized harmonic forcing. Spatio-temporal greylevel representation of $\operatorname{Re} \psi(x, t)$. Parameter settings are as in figure 2 , and for $t<0$ system is in state of natural oscillations tuned to $\omega_{0}^{c a}=0.4$ with a front at $X^{c a}=-1$. Forcing of frequency $\omega_{f}=1$ is applied at $X_{f}=-2$ and switched on at $t=0$ with different amplitudes $A_{f}$. (a) With $A_{f}=10^{-12}$, external forcing is unable to perturb nonlinear self-sustained structure. (b) With $A_{f}=10^{-11}$, spatial response reaches finite amplitude precisely at the front location $X_{n \ell}=X^{c a}$ and continuous competition between both frequencies takes place. (c) With $A_{f}=10^{-10}$, spatial response achieves nonlinear régime at $X_{n \ell}=-1.1$ upstream of $X^{c a}$ and rapidly replaces the natural oscillations.

if the upstream decaying front tail experiences an unperturbed medium. As soon as the front is overwhelmed by a finite-amplitude wave, the source of the 'steep' mode is suppressed and so is the entire self-sustained structure. The underlying AU region plays then no rôle in the dynamics since it is effectively masked by an 
externally imposed nonlinear wavetrain. Thus the oscillator-type behaviour of AU domains appears to be robust with respect to external forcing only if the strength of the forcing does not exceed an exponentially small level.

Effective control of the central AU domain requires that the forcing location and frequency are chosen so as to produce a downstream growing response, i.e., $k_{i}^{\ell+}\left(X_{f} ; \omega_{f}\right)<0$. This condition of downstream growth is fulfilled for a range of frequencies $\omega_{f}$ when forcing is applied at a position $X_{f}$ in the CU domain. Moreover, tuning to a single frequency can only be achieved when this forcing frequency produces nonlinear waves that are stable with respect to secondary perturbations. The precise range of possible control frequencies depends on the particular parameter settings of (2.1). In typical configurations, control of the system at twice or half the natural frequency is readily obtained.

In summary, an externally imposed nonlinear wave at the transition station from local convective to absolute instability entirely suppresses the intrinsic behaviour. Self-sustained oscillations may thus be controlled and tuned to a prescribed frequency, chosen such as to avoid damaging resonances or to improve performance of the system under consideration. Due to exponential growth of the forced response in the CU region, only an exponentially small forcing amplitude, and hence controller power, is required to achieve this result.

Enlightening and fruitful collaboration with Nigel Peake is greatfully acknowledged.

\section{References}

Babcock, K. L., Ahlers G. \& Cannel D. S. 1991 Noise-sustained structure in TaylorCouette flow with through flow. Phys. Rev. Lett. 67, 3388-3391.

Bouissou, P., Chiffaudel, A., Perrin, B. \& Tabeling, P. 1990 Dendritic side-branching forced by an extrenal flow. Europhys. Lett. 13, 89-94.

Bender, C. M. \& Orszag, S. A. 1978 Advanced mathematical methods for scientists and engineers. New York: McGraw-Hill.

Bers, A. 1983 Space-time evolution of plasma instabilities-absolute and convective. In Handbook of plasma physics (eds M. N. Rosenbluth \& R. Z. Sagdeev), pp. 451-517. Amsterdam: North-Holland.

Bewley, T. R. \& Liu, S. 1998 Optimal and robust control and estimation of linear paths to transition. J. Fluid Mech. 365, 305-349.

Briggs, R. J. 1964 Electron-stream interaction with plasmas. Cambridge (Mass.): M.I.T. Press.

Couairon, A. \& Chomaz, J.-M. 1996 Global instabilities in fully nonlinear systems. Phys. Rev. Lett. 77, 4015-4 018.

Couairon, A. \& Chomaz, J.-M. 1997a Absolute and convective instabilities, front velocities and global modes in nonlinear systems. Physica D 108, 236-276.

Couairon, A. \& Chomaz, J.-M. $1997 b$ Pattern selection in the presence of a cross flow. Phys. Rev. Lett. 79, 2666-2669.

Couairon, A. \& Chomaz, J.-M. 1999 Fully nonlinear global modes in slowly varying flows. Phys. Fluids 11, 3688-3 703.

Cross, M. C. \& Hohenberg, P. C. 1993 Pattern formation outside of equilibrium. Rev. Mod. Phys. 65, 851-1 112.

Dee, G. \& Langer, J. S. 1983 Propagating pattern selection. Phys. Rev. Lett. 50, 383-386.

Article submitted to Royal Society 
Ebert, U. \& Saarloos, W. van 2000 Front propagation into unstable states: universal algebraic convergence towards uniformly translating pulled fronts. Physica D 146, 199.

Ho, C.-M. \& Huerre, P. 1984 Perturbed free shear layers. Ann. Ref. Fluid Mech. 16, 365-424.

Huerre, P. \& Monkewitz, P. A. 1990 Local and global instabilities in spatially developing flows. Ann. Rev. Fluid Mech. 22, 473-537.

Huerre, P. 2000 Open shear flow instabilities. In Perspectives in Fluid Dynamics (eds G. K. Batchelor, H. K. Moffatt \& M. G. Worster), pp. 159-229. Cambridge: Cambridge University Press.

Kapral, R. \& Showalter, K. (eds) 1995 Chemical waves and patterns. Dordrecht: Kluwer.

Le Gal, P. \& Croquette, V. 2000 Visualization of the space-time impulse response of the subcritical wake of a cylinder Phys. Rev. E 62, 4424-4426.

Meunier, N., Proctor, M. R. E., Sokoloff, D. D., Soward, A. M. \& Tobias, S. M. 1997 Asymptotic properties of a nonlinear $\alpha \omega-$ dynamo wave: period, amplitude and latitude dependence. Geophys. Astrophys. Fluid Dynamics 86, 249-285.

Pier, B. \& Huerre, P. 2001 Nonlinear synchronization in open flows. J. Fluids Struct. 15, 471-480.

Pier, B., Huerre, P. \& Chomaz, J.-M. 2001 Bifurcation to fully nonlinear synchronized structures in slowly varying media. Physica $D$ 148, 49-96.

Reed, H. L. \& Saric, W. S. 1989 Stability of three-dimensional boundary layers. Ann. Rev. Fluid Mech. 21, 235-284.

Winfree, A. T. 1987 When time breaks down. Princeton: Princeton University Press. 